\title{
EL DEBATE SOBRE LA DESCENTRALIZACIÓN FORZADA DE LA BBC
}

Rodríguez-Castro, Marta ${ }^{1}$

Universidade de Santiago de Compostela, España

m.rodriguez.castro@usc.es

Material original autorizado para su primera publicación en la revista académica REDMARKA. Revista Digital de Marketing Aplicado.

https://doi.org/10.17979/redma.2018.01.020.4802

Recibido: 13 octubre 2017

Aceptado: 18 junio 2018

\section{Resumen}

Desde finales del siglo pasado, el Reino Unido ha experimentado un proceso de descentralización (devolution) mediante el cual las naciones que componen el Estado han recibido más competencias. La BBC, como estandarte de la radiodifusión de servicio público en el Reino Unido, y los medios de comunicación en general, han sido también objeto de debate en relación con esta descentralización. Frente a las demandas de las naciones de unos medios de comunicación públicos que representasen sus intereses y especificidades, los gobiernos de Westminster consideraron que ceder competencias en esta materia debilitaría a la BBC y minaría su posición en la sociedad británica. Sin embargo,

\footnotetext{
${ }^{1}$ Graduada en Comunicación Audiovisual por la Universidad de Santiago de Compostela (USC) y máster en Investigación aplicada a medios de comunicación por la Universidad Carlos III de Madrid. Actualmente es doctoranda en Comunicación e información contemporánea en la USC y disfruta de un contrato FPU. Su investigación se centra en el estudio comparativo de la televisión pública europea, ahondando en cuestiones de regulación, gobernanza y creación y gestión del valor público.
}

REDMARKA IMARKA-Universidad de A Coruña - CIECID 
este debate tuvo que ser abordado con mayor flexibilidad a raíz del referéndum para la independencia de Escocia que tuvo lugar en 2014, un punto de inflexión en la relación entre el gobierno central y las naciones, que tuvo repercusiones también en el proceso de renovación de la BBC Royal Charter en 2016. En 2017, la BBC anunciaba importantes inversiones en todas las naciones, entre las que destacaban las dirigidas a Escocia, por incluir la creación de un nuevo canal de televisión para BBC Scotland. A pesar de que el lanzamiento de este canal está previsto para la segunda mitad de 2018, esta propuesta tiene que superar un Public Interest Test en el que se pruebe que su valor público justifica su impacto de mercado. Este artículo aborda el proceso de descentralización forzada de la BBC desde los inicios de la devolution en el Reino Unido hasta la actualidad, analizando también el funcionamiento del Public Interest Test en contraste con su predecesor, el Public Value Test.

Palabras clave: BBC Scotland, Public Interest Test, medios de proximidad, televisión regional, descentralización, políticas de comunicación

\section{Abstract}

Since the last years of the past century, the United Kingdom has undergone a devolution process through which the nations that conform the State have received greater competencies. The $\mathrm{BBC}$, as the cornerstone of the British public service broadcasting system, and media in general, have also been the object of a debate around this devolution process. In the face of the nation's demands for public service media to represent their interest and specificities, Westminster has considered that to hand over competencies in this field would weaken the BBC and undermine its position within the British society. However, this debate had to be approached with greater flexibility as a consequence of the 2014 Scottish Independence Referendum, a turning point in the relation between the central government and the nations, which also impacted the renewal of the BBC Royal Charter in 2016. In 2017, the BBC announced major investments in the nations, specially in Scotland, as the plans of the corporation included a new television channel for BBC Scotland. Even though the launch of this channel is scheduled for the fall of 2018, first the proposal needs to pass a Public Interest Test which 
proves that its public value justifies its market impact. This paper approaches the forced devolution process of the BBC since the 1990s up to the present, also analyzing the functioning of the Public Interest Test in relation with its predecessor, the Public Value Test.

Keywords: BBC Scotland, Public Interest Test, proximity media, regional television, devolution, media policy.

\section{INTRODUCCIÓN}

Durante el primer cuatrimestre del pasado 2017, la BBC anunció las mayores inversiones en sus ramificaciones nacionales en Escocia, Gales e Irlanda del Norte de los últimos veinte años. Este aumento del presupuesto dedicado a las naciones se dirigía fundamentalmente a engrosar la producción y programación de contenidos diversos, que abarcasen tanto información como actualidad y entretenimiento, con el doble objetivo de conseguir una mejor representación nacional tanto para las propias naciones como para el conjunto del Reino Unido, además de desplazar la producción de contenidos fuera de Londres y repartirla por el conjunto del territorio británico, dinamizando así las industrias creativas de Escocia y Gales, principalmente.

El aparente reconocimiento de la BBC de la necesidad de aumentar la programación específica para las naciones ha tenido lugar tras la entrada en vigor de la nueva Royal Charter, el documento que determina la misión y enmarca la actividad de la BBC a largo plazo. Su proceso de renovación ha estado marcado por un contexto político convulso al que Schlesinger ha denominado "doble crisis de identidad" (Schlesinger, 2016), en referencia a la posición del Reino Unido con respecto a la Unión Europea y a la situación en Escocia tras el referéndum por la independencia. La situación política y las demandas sociales han dibujado el marco para la renovación de la BBC Charter, y lo que parece ser un primer paso hacia la descentralización de competencias dentro de la BBC. 
En la presente comunicación se trazará un recorrido a través de las demandas por una mayor cesión de competencias en materia de radiodifusión que se han sucedido en Reino Unido, focalizando la atención en el caso de Escocia. Tomamos como punto de partida el proceso de descentralización y devolución de competencias a las naciones (devolution) iniciado oficialmente en el año 1999, hasta llegar a la situación actual, marcada por la decisión de iniciar un Public Interes Test sobre la aprobación de un canal televisivo propio para BBC Scotland, pasando por el referéndum por la independencia de Escocia y por el debate en torno a la renovación de la Royal Charter.

\section{MARCO TEÓRICO}

En un contexto mediático marcado por la transnacionalización de la producción y la circulación interconectada de los contenidos, las televisiones públicas necesitan de un apoyo institucional contundente para poder, a su vez, ejercer de soporte y piedra angular de un mercado audiovisual nacional y/o regional (Chalaby, 2016). Dentro de esta ecología mediática global, los medios de comunicación de proximidad juegan un papel fundamental a la hora de conectar con comunidades e identidades de corte regional o local. El complemento "de proximidad" se ha acuñado para englobar "la polisemia de lo local con las nociones de territorio, identidad, pero también la complicidad entre emisores y audiencias y, muy importante, como marco competencial para el nuevo sistema audiovisual democrático" (de Moragas, 2015: 25).

En un estudio de 2016 sobre medios de comunicación regionales y locales, el Observatorio Audiovisual Europeo (OEA) incidía también en lo especial de esta relación entre audiencias y medios de proximidad "al tender la relación entre los medios regionales y las personas de las áreas y comunidades a las que sirven a ser más próximas que, por ejemplo, a niveles nacionales o internacionales" (OAE, 2016: 11), además de reconocer su papel fundamental en el la configuración de un espacio comunicativo para la expresión de las identidades regionales (OEA, 2016: 12). 
La misión de los medios de comunicación de proximidad en general, y de los que además son de carácter público, en particular, está ligada a la cobertura regional y local de información y temáticas que, de no existir este tipo de comunicaciones, contarían con un espacio muy limitado, si no inexistente, en medios nacionales o transnacionales. Es por ello que, tal y como apuntábamos antes, "en un contexto de globalización, los mecanismos comunicativos que permiten fortalecer las identidades más próximas al individuo adquieren una importancia destacada" (Manias-Muñoz y Muntsant, 2017: 192).

Sin embargo, la relevancia de los medios de comunicación de proximidad no siempre cuenta con el reconocimiento o la consideración suficientes y los de naturaleza pública suelen experimentar dificultades económicas notables, llegando incluso a la "neutralización de su misión pública de televisiones de identidad y proximidad" (Azurmendi, 2013: 356). Por otra parte, la articulación del sistema audiovisual, público y privado, difiere en cada país, por lo que mientras los medios de comunicación públicos alemanes, los belgas o los españoles construyen sus estructuras mediáticas sobre una base de proximidad, otros países, como Reino Unido, parten de un fuerte centralismo en su constitución y gobernanza, a pesar de que uno de los principios públicos que debe regir el funcionamiento de la BBC es precisamente "reflejar, representar y servir a las diversas comunidades de todas naciones y regiones del Reino Unido y, al mismo tiempo, apoyar la economía creativa del Reino Unido" [BBC, 2016: $6(4)]$.

\section{METODOLOGÍA}

El objetivo principal de esta comunicación es estudiar la evolución de la relación entre Escocia y la BBC como institución de corte centralista. A pesar de que en Gales e Irlanda del Norte también se han producido -y se siguen produciendotensiones con respecto al centralismo de la BBC, se ha seleccionado el caso concreto de Escocia por diferentes motivos. En primer lugar, se trata de la nación donde el debate en torno a la devolución de competencias en radiodifusión ha sido más intenso. Las elecciones al parlamento escocés de 2007 situaron por primera vez al Scottish National Party (SNP) en el gobierno, aumentando su

REDMARKA IMARKA-Universidad de A Coruña - CIECID

Año XI, Número 20, (2018), vol. 1 pp. 77-95

http://www.redmarka.net/ ISSN 1852-2300 
número de escaños en 2011 y haciendo posible la celebración de un referéndum por la independencia en 2014 que, finalmente, tuvo como resultado la permanencia en el Reino Unido. El movimiento independentista escocés volvió a poner sobre la mesa la cuestión de las competencias en políticas de comunicación y el papel de la BBC llegó a ser criticado por sectores nacionalistas alegando una cobertura informativa parcial a favor del No (Plunkett, 2014).

En estrecha relación con esta polémica, el caso de Escocia también merece atención por el hecho de que, entre los planes de aumento de la inversión para BBC Scotland se incluye la introducción de un nuevo canal de televisión propio que actualmente está siendo objeto de un Public Interest Test, la prueba de evaluación previa que sustituye al Public Value Test tras la entrada en vigor de la nueva Royal Charter. Esta coyuntura convierte a BBC Scotland en un objeto de estudio idóneo para ahondar en la relación entre la BBC y las naciones y regiones del Reino Unido.

La metodología que se ha empleado en esta investigación ha sido el análisis de documentos (Karppinen y Moe, 2012). Para dirigir el estudio hacia objetivos más concretos, se ha planteado la siguiente pregunta de investigación:

RQ. La creación de un canal de televisión para BBC Scotland, ¿va acompañada de una cierta descentralización de la gobernanza y de mayor cesión de control sobre la toma de decisiones a Escocia, tal y como se ha venido demandando en los últimos años?

\section{RESULTADOS}

\subsection{La devolución de competencias en el Reino Unido}

En el año 1997, el gobierno laborista impulsó la celebración de un referéndum sobre la devolución de poderes desde el Parlamento Británico hacia las naciones de Escocia y Gales. En ambos casos el resultado fue positivo, iniciándose así un proceso de descentralización y delegación de competencias hacia el Parlamento Escocés y la Asamblea Nacional de Gales. Irlanda del Norte cuenta con una compleja serie de antecedentes de descentralización y negociación con los 
gobiernos de Londres, y aunque la Asamblea de Irlanda del Norte, instaurada en 1998, fue suspendida en 2002, fue reestablecida cinco años más tarde. Inglaterra, por su parte, no cuenta con una cámara representativa propia, sino que todas las decisiones relativas a la política inglesa se toman desde Westminster.

Así, durante los últimos veinte años, el Reino Unido "ha pasado de ser un estado unitario a un sistema político complejo y multinivel, a través de un proceso de devolución asimétrica" (Keating, 2017: 1). Esto ha sido posible, en gran parte, al papel de la Unión Europea como aval del proceso, estableciendo un marco legal que añadía garantías a la descentralización, además de mediante la provisión de una serie de regulaciones que afectaban directamente a las competencias transferidas ${ }^{2}$.

La devolución no se ha realizado de forma homogénea en las tres naciones, por lo que en el Reino Unido coexisten distintos niveles de descentralización. En el caso de Escocia, el Scotland Act de 1998 reconoce como competencias del Parlamento Escocés la educación, la justicia o la agricultura, pero otros asuntos quedan reservados en exclusiva a Westminster, entre los que se encuentra la regulación y el control de los medios de comunicación (Hibberd, 2007). Según Schlesinger, Miller y Dinan (2001), la radiodifusión se mantuvo en un segundo plano durante estos primeros años de descentralización. Sin embargo, una demanda de mayor cesión de control en esta materia, fundamentalmente en relación al funcionamiento y la gobernanza de la BBC, comenzó a cobrar fuerza en Escocia a principios del nuevo siglo.

\subsection{Las primeras demandas de descentralización de los medios públicos británicos}

Tras su victoria en las elecciones al parlamento escocés de 2007 , el SNP se situó en el gobierno escocés y lideró la discusión en torno a la descentralización

\footnotetext{
${ }^{2}$ El papel que ha jugado la Unión Europea durante el proceso de devolución de poderes británico ha sido de tal importancia que la salida del Reino Unido de la UE abre un debate en torno a la viabilidad de la unión del estado británico, aumentando la tensión, en concreto, en relación a Escocia. Para más información, ver Keating, 2017.
}

REDMARKA IMARKA-Universidad de A Coruña - CIECID 
de la radiodifusión. Una de las primeras trasposiciones oficiales de estas demandas de reconfiguración del sistema audiovisual público del Reino Unido hacia el debate político fue la creación de la Scottish Broadcasting Commission (SBC) por parte del gobierno escocés en el año 2007, con el objetivo de realizar una investigación independiente sobre el estado de los medios de comunicación y de la producción televisiva en Escocia y de identificar además iniciativas que pudiesen impulsar las industrias creativas escocesas.

Los resultados de la investigación de la $\mathrm{SBC}$ se plasmaron en el informe "Platform for Success (2008). Tras ahondar en la situación de los medios de comunicación, el grupo de trabajo de la SBC señaló que, a pesar de lo beneficioso que resulta para Escocia formar parte de la red británica de producción y circulación de contenidos, la posición de esta nación en el marco del Reino Unido era marginal. Las recomendaciones de la SBC giraban en torno a una mayor inversión en la producción en Escocia por parte de las televisiones con misión servicio público británicas (la BBC, Channel 4 e ITV), mediante la revisión de sus políticas de subcontratación. A pesar de no recomendar la devolución de la competencia sobre radiodifusión, la SBC sí que incluyó entre sus propuestas para la dinamización de las industrias creativas escocesas la creación de un canal de televisión propio para Escocia, con su respectiva plataforma online. Este conjunto de medidas, junto con una mayor participación en la gobernanza y en la toma de decisiones de la BBC y de BBC Scotland, supondrían una mejora notable en la cobertura informativa sobre la nación, además de ampliar la oferta de contenidos en la que la realidad escocesa estuviese mejor representada.

El gobierno escocés abrazó el informe de la SBC e inició conversaciones con el gobierno británico, con Ofcom y con distintas radiotelevisiones, entre ellas la BBC (The Scottish Government, 2009), con resultados, en general, por debajo de lo esperado por parte del gobierno escocés. Además, antes de implantar los cambios deseados por Escocia, era necesario resolver ciertas cuestiones que se planteaban problemáticas: encontrar la forma de compatibilizar la gobernanza existente con una posible rendición de cuentas de la BBC ante el parlamento 
escocés; garantizar la independencia y la imparcialidad bajo el nuevo sistema o estudiar como afectarían dichos cambios a la recolección y distribución del canon son algunos de los problemas que detecta Schlesinger (2008) en ese momento concreto del debate, y que todavía hoy, habiendo conseguido ciertos avances, parecen no estar completamente resueltos.

\subsection{El referéndum para la independencia de Escocia de 2014}

EI SNP renovó su posición en el gobierno de Escocia en las elecciones de 2011 con el debate en torno a la descentralización de los medios de comunicación no solo sin resolver, sino todavía más candente. Una de las demandas más notables durante estos años, aunque presente desde décadas atrás, estaba relacionada con lo que se ha denominado "Scottish Six". BBC Scotland funciona como un centro regional de la BBC en Escocia, produciendo contenido para el conjunto de la cadena, así como para las audiencias escocesas. Lo que demandan los defensores del "Scottish Six" es una desconexión de la emisión general de BBC1 entre las 18:00 y las 19:00, es decir, durante la emisión del boletín informativo de más audiencia en el Reino Unido. Así, BBC Scotland se encargaría de elaborar su propio informativo bajo un prisma de proximidad, ofreciendo unos contenidos de mayor interés para la audiencia escocesa que la información centralista del informativo producido desde los estudios centrales de la BBC en Londres. Esta idea no fue bien recibida ni por los sucesivos gobiernos británicos ni por la BBC, al considerar que el "Scottish Six" sería el primer paso hacia una BBC federal y débil (Birt, 2003, en Hibberd, 2007) o que podría potenciar el sentimiento independentista (Schlesinger, 2008).

La segunda legislatura del SNP supuso un punto de inflexión dentro del debate sobre la devolución de competencias, debido al impulso y a la celebración del referéndum para la independencia de Escocia. Este acontecimiento intensificó la tensión entre Escocia y la BBC, que llegó a ser acusada de cobertura parcial a favor de la unidad del Reino Unido, a pesar de que estudios como el de Dekavalla y Jelen-Sanchez (2016) argumentan que los representantes de ambas opciones contaron con un espacio similar en la información relativa al referéndum en el programa "Reporting Scotland".

REDMARKA IMARKA-Universidad de A Coruña - CIECID 
A pesar de que los ciudadanos escoceses votaron por permanecer en el Reino Unido (los resultados fueron un $44,7 \%$ para el Sí y un 55,3\% para el No a la independencia), el referéndum supuso un cambio en la relación entre los poderes escoceses y el Gobierno británico. Se abrió así una puerta a la negociación sobre la cesión de mayor poder de decisión a Escocia en distintas competencias ${ }^{3}$, entre las que esta vez se hacía mención el Gaelic Media Service, aunque de forma muy reducida. Este cambio de actitud coincidió con el proceso de debate sobre la renovación de la Royal Charter de la BBC, delimitándose esta vez el papel que jugaría Escocia en la delineación y aprobación del texto final en el Memorandum of Understanding (Smith Commission, 2015). Este acuerdo, firmado por el Department for Culture, Media and Sport (DCMS), la BBC y el gobierno y parlamento escoceses en 2015, garantizaba la participación de las instituciones escocesas en el proceso de elaboración de la nueva Royal Charter y comprometía a la BBC a presentar sus informes anuales ante Escocia y a rendir cuentas ante el parlamento escocés. Además, todas estas condiciones serían incluidas de forma oficial en la nueva Royal Charter.

\subsection{El impacto del debate sobre la descentralización de la radiodifusión en la Royal Charter de 2016-2027}

Al impacto directo de la celebración del referéndum hay que sumar otro factor que forzó el aumento de la participación de las instituciones escocesas en la renovación de la Royal Charter: una cierta deficiencia de la corporación en cuanto a la representación y participación de las naciones y regiones británicas. Según la encuesta encargada por el BBC Trust en 2016 sobre el desempeño de la $\mathrm{BBC}$ en relación a sus propósitos públicos, el relacionado con las naciones, regiones y comunidades británicas es el que peor resultados obtiene, en línea con los datos de años previos (ICM, 2016: 38): tan solo el 51\% de los encuestados valoran los resultados de la BBC en relación con este propósito público de forma positiva.

\footnotetext{
${ }^{3} \mathrm{El}$ incremento de competencias que fueron devueltas a Escocia se plasmó en el Scotland Act de 2016.
}

REDMARKA IMARKA-Universidad de A Coruña - CIECID 
El desglose del propósito público en categorías dirige la atención a la cuestión relacionada con la representación de las distintas naciones y regiones dentro del contexto de conjunto del Reino Unido, tal y como se recoge en el Gráfico 1. A pesar de las ligeras mejoras en casi todos los indicadores, la diferencia entre lo que los ciudadanos esperan de la BBC y los resultados que perciben todavía distaban de lo deseado por la corporación.

Gráfico 1: Desempeño del propósito "Naciones, regiones y comunidades” por categorías

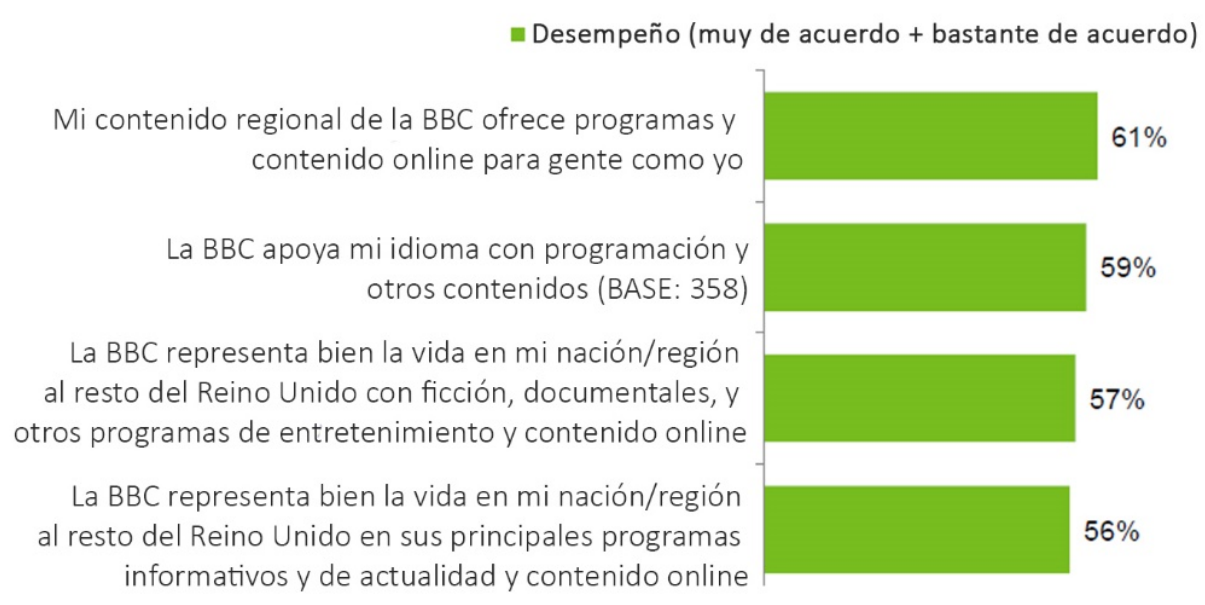

Cambio

2015

$+2$

$-2$

$+2$

$+2$

Fuente: $\operatorname{ICM}(2016: 39)$

Otro informe de ese mismo año, esta vez a cargo de Goldsmiths, de la University of London, dedicaba un capítulo a analizar la situación de las radiotelevisiones públicas británicas en su relación con las naciones y regiones en un contexto de cambio constitucional y aumento de la presión descentralizadora en el Reino Unido. La idea central del informe apunta a que las bajas inversiones en contenido producido fuera del área londinense afectaron notablemente a la reputación de las emisoras públicas entre las audiencias nacionales y regionales, alimentando una concepción centralista de un sistema televisivo que presta poca atención a la diversidad del país (Goldsmiths, 2016: 118). Los esfuerzos de dispersión de la producción realizados desde 2008 han supuesto resultados modestos $-\mathrm{y}$, en muchas ocasiones, camuflados ${ }^{4}$-, que además no se han

\footnotetext{
${ }^{4}$ Son muchas las productoras afincadas en Londres que se desplazan fuera de la capital para la producción de contenido con el objetivo de cumplir la cuota de descentralización exigida, para volver después a su base
}

REDMARKA IMARKA-Universidad de A Coruña - CIECID 
acompañado de la descentralización de la toma de decisiones, que sigue produciéndose en Londres.

En este contexto, las demandas de las instituciones escocesas dentro del proceso de renovación de la Royal Charter de la BBC se estructuraron en torno a tres puntos básicos: la mejora de la oferta de BBC Scotland, con un contenido más representativo; una gobernanza y una estructura de la BBC más receptivas y descentralizadas; y prácticas de producción y subcontratación que apoyasen las industrias creativas escocesas (The Scottish Government, 2016). Tras la creación de una comisión de expertos que abordó la cuestión de la radiodifusión ante el parlamento escocés, y la comparecencia del propio director general de la BBC, Tony Hall, ante dicha institución, la BBC reconoció sus deficiencias en el servicio a los ciudadanos escoceses y se propuso rectificarlas en la etapa que la nueva Royal Charter inauguraría en 2017.

De esta forma, en el texto definitivo de la actual Royal Charter, que estará vigente hasta fin de 2027, mantiene como propósito público la representación de las naciones, regiones y comunidades británicas, y extiende la gobernanza y la rendición de cuentas a las instituciones descentralizadas. En el ámbito de la gobernanza, el nuevo órgano de dirección centralizada, el BBC Board, incluye ahora a cuatro representantes nacionales, uno por cada una de las Naciones británicas, en el cargo de consejeros no ejecutivos. En la anterior Royal Charter se contemplaba una representación similar dentro del BBC Trust, pero la designación de estos consejeros no ejecutivos del BBC Board es una novedad del vigente texto.

En cuanto a la rendición de cuentas, los planes e informes anuales deben hacer referencia a la oferta para las naciones y las regiones, debiendo además el informe anual informar sobre el impacto del propósito creativo de la BBC en los distintos territorios. La nueva Charter establece que la BBC deberá entregar su informe anual a los ministros escoceses, galeses y norirlandeses el mismo día

original. Esta lógica del "lift and shift" no genera la riqueza esperada ni el impulso de las industrias creativas de las distintas naciones y regiones. 
que lo reciban el Parlamento Británico y la Ofcom. De así requerirlo, la BBC deberá comparecer ante el Parlamento Escocés, la Asamblea Nacional de Gales o la Asamblea de Irlanda del Norte para presentar sus informes anuales, las cuentas del grupo y la auditoría de estas. Además, se recoge la participación de las instituciones descentralizadas en futuros procesos de revisión de la Royal Charter, tal y como se estipulaba en el anteriormente referenciado Memorandum of Understanding.

La entrada en vigor de la nueva Royal Charter fue acompañada por el anuncio de importantes inversiones de la corporación pública en las naciones británicas, tal y como se apuntaba al inicio de esta comunicación. A pesar de que la BBC reforzaría su servicio en Irlanda del Norte (11 millones de libras) y Gales (8,5 millones de libras), fue en Escocia donde anunció un mayor aumento de recursos (20 millones de libras), además de atender a una de las demandas escocesas más recurrentes de las dos últimas décadas: el impulso de un canal propio para BBC Scotland.

\subsection{El Public Interest Test de BBC Scotland}

La Royal Charter vigente establece que cualquier cambio material que desee introducir la BBC debe ser objeto de un Public Interest Test en el que la propuesta se evalúe desde el punto de vista de su valor público y de su impacto en el mercado y la competencia. La anterior Royal Charter había introducido este tipo de mecanismo, pionero en Europa (Donders y Moe, 2011), bajo el nombre de Public Value Test. Aunque ambas pruebas comparten una misma filosofía, lo cierto es que la introducción del Public Interest Test viene acompañada de una serie de cambios en el desarrollo de este procedimiento de evaluación previa.

La primera gran diferencia entre ambas pruebas radica en los organismos encargados de llevarlas a cabo. El Public Value Test se organizaba desde el BBC Trust, involucrando al BBC y a la Ofcom, pero la nueva Royal Charter extingue dicho organismo de gobernanza, haciéndose necesaria una reestructuración de las competencias para el Public Interest Test. Así, la Ofcom, 
como autoridad supervisora independiente de la BBC, es ahora la responsable del desarrollo de la prueba y de la decisión final de la propuesta.

El segundo cambio de mayor calado entre las pruebas es el incremento de la complejidad del proceso, que, a efectos prácticos, debe realizarse ahora por duplicado. La nueva normativa sobre el Public Interest Test (PIT) establece que la BBC deberá completar una primera versión de la prueba antes de que la Ofcom tome el control del proceso y aplique de nuevo el procedimiento sobre la propuesta. Solo si la BBC, a partir de su primer análisis, constata la creación de valor público del servicio propuesto, la reducción de su posible impacto adverso sobre el mercado y la competencia a su mínima expresión, y la justificación de dicho impacto por su valor público, iniciará el procedimiento la Ofcom.

A pesar de que la normativa establece dos modalidades para la realización del PIT por parte de la Ofcom, el BBC Competition Assessment (BCA), de una duración de hasta seis meses, el shorter competition assessment, con una duración inferior para aquellos casos en los que la BBC haya realizado un PIT minucioso y completo; el nuevo proceso parece incidir en una cierta redundancia y duplicación de trabajo que, no obstante, puede justificarse por la necesidad de contrastar dos informes de diferente procedencia para garantizar la correcta valoración del conjunto de variables cuantitativas y cualitativas que componen la prueba.

La creación de un canal de televisión para BBC ha sido la propuesta encargada de inaugurar el PIT. El anuncio de la BBC de un aumento de la inversión en Escocia incluía el establecimiento de un nuevo canal para BBC Scotland con emisión diaria desde las 19:00 hasta medianoche y con el objetivo de reflejar mejor a Escocia y a su cultura, dentro y fuera de sus barreras. La propuesta de la BBC necesita de la concesión de una nueva licencia para llevarse a cabo y, al constituir un cambio material dentro de los servicios de la corporación, debe superar un PIT para llevarse a cabo.

Así, tras el anuncio de la BBC en febrero de 2017, se inició la primera fase del proceso, en la que la propia BBC se encarga de reunir las evidencias necesarias 
para evaluar el valor público y el impacto de mercado de su propuesta. Tras realizar varias reuniones con distintos grupos de interés, convocar una consulta pública entre junio y julio de ese mismo año y encargar sendos informes a Pinto Addley Price Associates (PAPA, cualitativo) y a Kantar TNS (cuantitativo), la BBC publicaba en noviembre las conclusiones de su primer PIT: el valor público de un canal para BBC Scotland se demostró alto y su impacto adverso sobre el mercado y la competencia, ya en un principio poco probable, se redujo todavía más modificando ciertos aspectos de la propuesta inicial, como la limitación de la emisión fuera del horario de 19:00 a 00:00 a 150 horas anuales. La sugerencia de la BBC, por tanto, fue la aprobación de su propuesta.

Concluida esta primera fase, la Ofcom inició su propia consulta pública (noviembre-diciembre 2018) y, tras revisar el PIT de la BBC, optó por llevar a cabo un BCA, es decir, la modalidad más minuciosa y duradera de PIT, que concluirá en julio de 2018 con la decisión final de la autoridad independiente sobre la aprobación o no de la propuesta de la BBC. De contar con luz verde para la concesión de la licencia, la fecha establecida para el lanzamiento del canal de BBC Scotland está programada para otoño de 2018.

\section{DISCUSIÓN Y CONCLUSIONES}

Las demandas por una mayor descentralización de los medios de comunicación, públicos y privados, han pasado de ser descartadas por parte de los gobiernos centrales y de los propios organismos de radiodifusión por considerarlas dañinas tanto para el sistema de comunicaciones como para el sistema político, a ser consideradas en el debate reciente a raíz, principalmente, del auge del nacionalismo escocés y de las negociaciones posteriores a la celebración del referéndum por la independencia de Escocia.

El análisis que se ha realizado por el transcurso de este debate político ha puesto de manifiesto el valor de unos medios de comunicación de proximidad que puedan estructural el espacio comunicativo de identidades y comunidades locales. Las críticas dirigidas hacia el centralismo de la BBC y las demandas de contenido tanto informativo como de entretenimiento en el que la sociedad 
escocesa pudiera reconocerse han sido claves en la nueva configuración de la corporación pública, plasmada en la Royal Charter vigente desde 2017.

Sin embargo, a la hora de responder a la pregunta de investigación que se planteaba en esta comunicación, concluimos que el aumento de la inversión en las naciones o incluso la creación de un canal de televisión propio para BBC Scotland distan de forma considerable de las ambiciones descentralizadoras que algunos sectores políticos y sociales tenían en mente para la BBC. Las inversiones en Gales e Irlanda del Norte han sido mucho más modestas, por lo que estos movimientos estratégicos por parte de la corporación pública parecen ser más un intento de controlar las presiones de los nacionalistas escoceses que una voluntad real de replantearse su papel en las naciones y regiones del Reino Unido.

La representación de las naciones en el BBC Board, a través de sus respectivos consejeros no ejecutivos, supone una continuación con la anterior gobernanza, en la que el BBC Trust también contaba con un representante de cada nación. En el ámbito de la rendición de cuentas, sin embargo, se percibe un mayor avance, al oficializarse la comparecencia de la BBC ante las instituciones descentralizadas y crearse así una nueva vía para la negociación de cuestiones relacionadas con las líneas editoriales, de contenido y de producción de la corporación.

El Public Interest Test que está siendo aplicado a la propuesta de creación de un canal de televisión para BBC Scotland constituye un objeto de estudio a seguir con atención en investigaciones futuras. Una vez finalizado y publicado el informe final, se posibilitará un estudio comparativo completo entre el PIT y su antecesor, el Public Value Test, lo que también permitirá profundizar en los motivos detrás del cambio en este procedimiento de evaluación previa.

\section{RECONOCIMIENTOS DE LA INVESTIGACIÓN}

Los resultados de este artículo corresponden al proyecto de "Indicadores de gobernanza, financiación, rendición de cuentas, innovación, calidad y servicio público de las RTV europeas aplicables a España en el contexto digital"

REDMARKA IMARKA-Universidad de A Coruña - CIECID 
(Referencia CSO2015-66543-P) del Programa estatal de Fomento de la Investigación Científica y Técnica de Excelencia, subprograma estatal de Generación de Conocimiento del Ministerio de Economía y Competitividad de España, cofinanciado por el Fondo Europeo de Desarrollo Regional (FEDER) de la Unión Europea. Así como de la actividad de la Red Internacional de Investigación de Gestión de la Comunicación (REDES 2016 G-1641 XESCOM) apoyada por la Consellería de Cultura, Educación e Ordenación Universitaria de la Xunta de Galicia (referencia ED341D R2016/019). La autora Marta Rodríguez Castro es beneficiaria del Programa de Formación del Profesorado Universitario (FPU16/05234) financiado por el Ministerio de Educación, Cultura y Deporte (Gobierno de España).

\section{REFERENCIAS BIBLIOGRÁFICAS}

Azurmendi, A. (2013). Reformas de la televisión regional europea con lengua propia en el contexto actual de crisis. Revista Latina de Comunicación Social, 68, 355 - 382. DOI: https://doi.org/10.4185/RLCS-2013-981.

BBC (2016). Royal Charter for The Continuance of the British Broadcasting Corporation. Recuperado de: https://goo.gl/dwo3NG.

Birt, J. (2003). The Harder Path, the Autobiography. Londres: Time Warner.

Chalaby,J. K. (2016). Broadcasting policy in the era of global value chain-oriented industrialisation. Comunicación enviada a la consulta 'A Future for Public Service Television: Content and Platforms in a Digital World'. Recuperado de:

https://goo.gl/ rMMfYm.

de Moragas, M. (2015). La comunicación de proximidad 30 años después. En A. Nerekan Umaran, M.A. Casado del Río, R. Zallo Elgezabal y J.C. Miguel de Bustos (Eds.), Comunicación de proximidad. Cada vez más lejos. Marco, experiencias y regulación (21-37). Bilbao: Universidad del País Vasco. 
Dekavalla, M., y Jelen-Sanchez, A. (2016). Whose voices are heard in the news? A study of sources in television coverage of the Scottish independence referendum. British Politics, 12(4), 449-472. DOI: https://doi.org/10.1057/s41293-016-0026-4.

Donders, K., y Moe, H. (Eds.). (2011). Exporting the public value test: the regulation of public broadcasters' new media services across Europe. Gotemburgo: Nordicom.

Goldsmiths (2016). A future for Public Service Television. Content and Platforms in a digital world. Londres: University of London.

Hibberd, L. (2007). Devolution in Policy and Practice: A Study of River City and BBC Scotland. Westminster Papers in Communication and Culture 4(3), 107-125.

ICM Unlimited (2016). Purpuse Remit Survey Autumn 2016. UK Report.

Karpinnen, K. y Moe, H. (2012). What We Talk about When We Talk about Document Analysis. En N. Just y M. Puppis (Eds.), Trends in Communication Policy Research (175-193). Bristol: Intellect.

Keating, M. (2017). Brexit and Devolution in the United Kingdom. Politics and Governance, 5(2), 1-3.

Manias-Muñoz, M. \& Muntsant, A. M. (2017). BBC Alba: Servicio público y representación gaélica en escocia. Historia y Comunicación Social, 22(1), 191-206.

OAE (2016). Regional and local broadcasting in Europe. Estrasburgo: Observatorio Audiovisual Europeo.

Plunkett, J. (2014, 2 de junio). BBC's Scottish Independence coverage accused of pro-union bias. The Guardian. Recuperado de: https://goo.gl/4yfHVt.

SBC (2008). Platform for Success. Final Report of the Scottish Broadcasting Commission. Edimburgo: The Scottish Government. 
Schlesinger, P. (2016). "Whiter public service broadcasting in Scotland?", en Media Policy Project Blog, London School, Enero de 2016: https://goo.gl/6jHAzd.

Schlesinger, P. (2008). Broadcasting Policy and the Scottish Question. En T. Gardham, y D.A.L. Levy (Eds.), The Price of Plurality: Choice, Diversity and Broadcasting Institutions in the Digital Age (155-161). Oxford: Reuters Institute for the Study of Journalism.

Schlesinger, P., Miller, D. y Dinan, W. (2001). Open Scotland? Journalists, Spin Doctors and Lobbyist. Edimburgo: Polygon.

Smith Commission (2015). Memorandum of Understanding. Recuperado de:

https://

goo.gl/4GiZXJ.

The Scottish Government (2016). Scottish Government Policy paper - BBC Charter Renewal. Edimburgo: Scottish Government. ISBN: 978-1-78652457-7.

The Scottish Government (2009). Platform for Success: the Scottish Broadcasting Commission Report. Response from the Scottish Government. Edimburgo: The Scottish Government. 